\title{
Modeling motor connectivity using TMS/PET and structural equation modeling
}

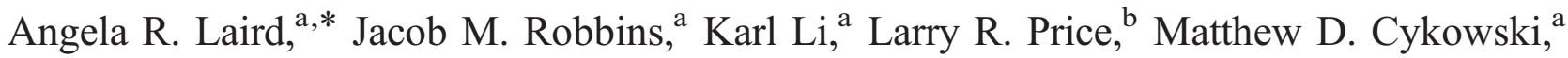 \\ Shalini Narayana, ${ }^{a}$ Robert W. Laird, ${ }^{c}$ Crystal Franklin, a and Peter T. Fox ${ }^{\text {a }}$ \\ ${ }^{a}$ Research Imaging Center, University of Texas Health Science Center, San Antonio, Texas, USA \\ ${ }^{\mathrm{b}}$ Colleges of Education and Science, Texas State University, San Marcos, Texas, USA \\ ${ }^{\mathrm{c}}$ Department of Physics, Texas Lutheran University, Seguin, Texas, USA
}

Received 6 August 2007; revised 14 January 2008; accepted 30 January 2008

Available online 15 February 2008

\begin{abstract}
Structural equation modeling (SEM) was applied to positron emission tomographic (PET) images acquired during transcranial magnetic stimulation (TMS) of the primary motor cortex $\left(\mathrm{M1}_{\text {hand }}\right)$. TMS was applied across a range of intensities, and responses both at the stimulation site and remotely connected brain regions covaried with stimulus intensity. Regions of interest (ROIs) were identified through an activation likelihood estimation (ALE) meta-analysis of TMS studies. That these ROIs represented the network engaged by motor planning and execution was confirmed by an ALE meta-analysis of finger movement studies. Rather than postulate connections in the form of an a priori model (confirmatory approach), effective connectivity models were developed using a model-generating strategy based on improving tentatively specified models. This strategy exploited the experimentally imposed causal relations: (1) that response variations were caused by stimulation variations, (2) that stimulation was unidirectionally applied to the $\mathrm{M} 1_{\text {hand }}$ region, and (3) that remote effects must be caused, either directly or indirectly, by the $\mathrm{M1}_{\text {hand }}$ excitation. The path model thus derived exhibited an exceptional level of goodness $\left(\chi^{2}=22.150, d f=38\right.$, $P=0.981, T L I=1.0)$. The regions and connections derived were in good agreement with the known anatomy of the human and primate motor system. The model-generating SEM strategy thus proved highly effective and successfully identified a complex set of causal relationships of motor connectivity.
\end{abstract}

(C) 2008 Elsevier Inc. All rights reserved.

Keywords: Transcranial magnetic stimulation; TMS; Motor; Structural equation modeling; SEM; Path analysis; Effective connectivity; Activation likelihood estimation; ALE; Meta-analysis

\footnotetext{
* Corresponding author. Research Imaging Center, University of Texas Health Science Center San Antonio, 7703 Floyd Curl Drive, San Antonio, TX 78229-3900, USA. Fax: +1 2105678152.

E-mail address: lairda@uthscsa.edu (A.R. Laird).

Available online on ScienceDirect (www.sciencedirect.com).
}

\section{Introduction}

Structural equation modeling (SEM) is a powerful, generalpurpose tool for statistical analysis and modeling of interactions between observed and unobserved (latent) variables (Schumacker and Lomax, 2004), with the typical goal of testing causal relationships among variables. In the field of brain imaging, SEM can be used to model distributed neural systems composed of multiple regions, with these regions being modeled as observed variables and the neural pathways connecting them being modeled as causal relationships (i.e., inter-regional covariances) (McIntosh et al., 1994; McIntosh and Gonzales-Lima, 1994). Because covariance analyses result in bidirectional paths, a substantial challenge for SEM in neuroimaging is to reduce these to unidirectional paths. The most common strategy is hypothesis-based, in which optional paths are constrained with a priori information, typically from anatomical or functional connectivity studies in human or nonhuman species. However, it is often difficult to defend the assumptions made when constructing these hypothesis-based models. Moreover, the number and location of regions in the network needs to be established such that critical nodes are not omitted, while at the same time excluding regions of no relevance. This is critical given that SEM procedures mandate a limited number of regions of interest; thus, the selection of ROIs can be open to experimenter bias. A further challenge is that once the regions are selected, restrictions must be made on the paths connecting those regions. Testing a model in which every region is bidirectionally connected to every other region, known as the saturated model (Schumacher and Lomax, 2004), is not a viable option unless the model contains a very small number of regions. The goal of SEM in any context is to achieve a parsimonious model (i.e., one with few path tracings), yet one that also exhibits an increase in evaluating the adequacy (e.g., goodness) of the model. It must be determined which connections should be omitted in the saturated model, then if the remaining connections are bidirectional or unidirectional. 
Recent studies have attempted to address these questions by developing methods to constrain starting models prior to SEM (Patel et al., 2006; Zheng and Rajapakse, 2006). The purpose of these methods is to analyze the structure of the data in order to determine strong relationships that can later be modeled using SEM in a confirmatory approach. However, SEM theory provides an alternate way of analyzing covariance structure between variables, one that specifies tentative models that best fit the given data (Hershberger et al., 2003). Exploratory SEM methods have been utilized in previous studies investigating bimanual coordination (Zhuang et al., 2005), tactile shape perception (Peltier et al., 2007), and to some extent, semantic decision-making with covert articulation (Bullmore et al., 2000). These studies restricted their analyses to a very small number of brain regions and/or imposed initial constraints on various possible paths. Here, the goal was to investigate the applicability of exploratory structural equation modeling in positron emission tomography (PET) data during transcranial magnetic stimulation (TMS) with a large number of neural regions and no assumed constraints of the initial model.

TMS has been used in conjunction with imaging methods such as PET (Fox et al., 1997; Paus et al., 1997) and functional magnetic resonance imaging (fMRI) (Bohning et al., 1997), as well as eventrelated potentials (ERP) (Ilmoniemi et al., 1997). TMS can be applied to a discrete cortical site and propagates orthodromically from that site, resulting in detectable changes in cerebral blood flow of the entire network of connected regions (Fox et al., 1997). This basic principle can be applied to SEM to generate unidirectional starting paths from which to model, which is the strategy used in this study. This novel strategy is possible due to the ability to draw a known starting path based on the given experimental design, an exclusive property of applying SEM to TMS/PET data. By their nature, functional imaging tasks in which activations are derived from various perceptual, motor, or cognitive events do not fall into this same category of design. In these tasks, the entire neural network is activated as a whole and the starting point of stimulation of the network can only be derived hypothetically. Thus, TMS is unique since a discrete cortical area of interest is directly stimulated and the delivered signal propagates throughout the connected network of interest.

In this study, path analysis, a form of SEM involving only observed variables, was used to model the connectivity of the TMSstimulated hand region of the left primary motor cortex $\left(\mathrm{LMI}_{\text {hand }}\right)$ in seven subjects. To reduce bias and minimize assumptions, regions in the $\mathrm{LMI}_{\text {hand }}$ TMS network of these subjects were restricted to those identified with activation likelihood estimation (ALE) meta-analysis of the TMS literature (Turkeltaub et al., 2002; Laird et al., 2005a). For each subject, TMS was delivered in a graded manner, resulting in graded responses both at the stimulation site and beyond. Thus, parametric intensity stimulation of the $\mathrm{LMI}_{\text {hand }}$ site occurred, ranging from above to below subjects' individual motor thresholds. This experimental design allowed for an SEM analysis that included: (1) the stimuli itself as a quantitative variable and (2) a known starting point for exploratory modeling of the system as a whole.

\section{Methods}

\section{Subjects}

Seven healthy, right-handed volunteers were included in this study ( 4 women, 3 men; mean age $=35$ years; standard deviation= 11.34 years; age range $=22-43$ years). All subjects gave their written, informed consent in accordance with the Declaration of Helsinki and with approval from the Institutional Review Board and Radiation Safety Committee of the University of Texas Health Science Center at San Antonio. All subjects were healthy (no medical, neurological, or psychiatric illnesses) and taking no medications. The use of TMS at $3 \mathrm{~Hz}$ was approved by the United States Food and Drug Administration (IDE K905059D, held by Peter Fox). The data acquired in these 7 subjects were used in prior publications (Fox et al., 2004, 2006), and consisted of subjects representing the group of "cortical responders." This designation indicated that significant PET activation during TMS was observed in these subjects. Four other subjects were omitted from the present analysis, as no significant PET activation was observed in this data, despite the presence of physiological evidence of stimulation.

\section{TMS/PET procedures}

TMS was delivered at $3 \mathrm{~Hz}$ via a robotic, image-guided system (Lancaster et al., 2004) to $\mathrm{LMI}_{\text {hand }}$. TMS was delivered with a watercooled, B-shaped coil (Cadwell, Kennewick, WA). The coil was powered by a Cadwell HSMS unit, which delivers a tri-phasic electric pulse with a total duration of $240 \mu$ s and a peak E-field of $435 \mathrm{~V} / \mathrm{m}$ at the coil surface at $100 \%$ of machine output. Motor thresholds were determined prior to imaging as described in Fox et al. (1997). During PET, each subject underwent two trials of TMS at 3 intensities relative to motor threshold (75\% MT, 100\% MT, and $125 \% \mathrm{MT}$ ), delivered in a pseudo-randomized order (6 stimulated scans per subject). TMS commenced $120 \mathrm{~s}$ prior to tracer injection and continued through the first $40 \mathrm{~s}$ of a 90 -s image acquisition. In addition, subjects were also imaged during two trials of rest $(0 \%$ MT).

PET image acquisition was performed using a General Electric (Milwaukee, WI) 4096 whole-body camera (pixel spacing=2 mm, spatial resolution $=6.5 \mathrm{~mm}$ FWHM in the axial plane, inter-plane center-to-center distance $=6.5 \mathrm{~mm}$, scan planes $=15, z$-axis $\mathrm{FOV}=$ $10 \mathrm{~cm}$ ). Brain blood flow was measured using $\mathrm{H}_{2}^{15} \mathrm{O}$, administered as an intravenous bolus of 8-10 $\mathrm{ml}$ saline containing $50-70 \mathrm{mCi}$ per injection, tailored to obtain satisfactory coincidence detection rates. Ninety seconds of data were acquired, triggered by the tracer entering the brain. Throughout the PET session, the subjects' heads were immobilized with an individually fitted, thermoplastic facial mask (Fox and Raichle, 1984). To minimize auditory activation due to the sound emitted by the TMS, foam earplugs were worn throughout the imaging study. Electromyographic (EMG) recordings were monitored throughout the period of TMS delivery to ensure effective stimulus delivery. Anatomical MRI was acquired in each subject and used to optimize spatial normalization. MR imaging was performed on a $1.9 \mathrm{~T}$ GE/Elscint Prestige (Haifa, Israel) at a voxel size of $1 \mathrm{~mm}^{3}$.

\section{Image pre-processing}

Images were reconstructed into 60 slices ( $2 \mathrm{~mm}$ thickness), with an image matrix size of $60 \times 128 \times 128$, using a $5 \mathrm{~mm}$ Hanning filter resulting in images with a spatial resolution of approximately $7 \mathrm{~mm}$ (FWHM). PET images were value-normalized to a whole-brain mean of 1000. PET and MRI data were co-registered using the Convex Hull algorithm (Lancaster et al., 1999). MRI data were spatially normalized in series using both the Spatial Normalization (SN) (Lancaster et al., 1995) and Octree SN (OSN) algorithms (Kochunov et al., 1999). The SN algorithm performed "global" (nine-parameter) spatial normalization, which registered each subject to the target shape provided by 
Table 1

Input data for ALE meta-analysis of $\mathrm{LMI}_{\text {hand }}$ TMS studies

\begin{tabular}{lrllll}
\hline Author & $N$ & Contrast & $\begin{array}{l}\text { Intensity } \\
(\% \mathrm{MT})\end{array}$ & $\begin{array}{l}\text { Frequency } \\
(\mathrm{Hz})\end{array}$ & Modality \\
\hline Bestmann & 12 & $\begin{array}{l}\text { Suprathreshold } \\
\text { rTMS - Rest }\end{array}$ & $110 \%$ & 3.125 & 3 T MRI \\
Bestmann & 12 & $\begin{array}{l}\text { Subthreshold } \\
\text { rTMS - Rest }\end{array}$ & $90 \%$ & 3.125 & 3 T MRI \\
Denslow & 11 & $\begin{array}{l}\text { TMS vs. Rest, } \\
\text { Increases }\end{array}$ & $110 \%$ & 1.0 & 1.5 T MRI \\
Siebner, 1999 & 12 & $\begin{array}{l}\text { rTMS vs. Rest } \\
\text { Siebner, 2000 }\end{array}$ & $\begin{array}{l}\text { Post-rTMS vs. } \\
\text { "just below" }\end{array}$ & 5.0 & ${ }^{18}$ FDG PET \\
Speer & $10 \begin{array}{l}\text { Rest } \\
\text { Positive } \\
\text { correlations }\end{array}$ & $80 \%-120 \%$ & 1.0 & $\mathrm{H}_{2}^{15}$ O PET \\
\hline
\end{tabular}

5 papers with 6 contrasts and 53 foci were included in the TMS metaanalysis (listed in alphabetical order by first author).

$n=$ number of subjects included in a given study; Intensity (\% MT)= intensity at which TMS was delivered relative to each individual's relative percent of motor threshold.

the Talairach and Tournoux (1988) atlas. The OSN algorithm performed "local" spatial normalization, in which each subject's brain was anatomically deformed to match the median of the group in a high-resolution $\left(1 \mathrm{~mm}^{3}\right)$, fully $3 \mathrm{D}$ manner. OSN processing was used to optimize registration of anatomical features (and thereby functional areas) across subjects. OSN-derived deformation fields were applied to the PET data prior to computation of statistical parametric images (SPIs).

\section{ALE meta-analysis}

An aim of this study was to develop more standardized, less userdependent methods of ROI selection prior to SEM analyses. As an alternative to the highly variable methods currently observed in the neuroimaging literature, the ROI selection procedure was guided by a quantitative, voxel-based meta-analysis of TMS studies.

\section{LMI hand TMS literature search}

A PubMed literature search was carried out to identify all studies published prior to June 2006 in which repetitive TMS (rTMS) was used to stimulate the left primary motor cortex during fMRI or PET $\left(\mathrm{H}_{2}^{15} \mathrm{O}\right.$ or $\left.{ }^{18} \mathrm{FDG}\right)$ imaging. The search results were filtered to eliminate studies that lacked whole-brain results in the form of stereotactic $(x, y, z)$ coordinates, which did not apply TMS concurrently with tracer uptake (PET) or with imaging (fMRI), and which had fewer than five subjects. Studies were also excluded if they reported only individual subject data, data from non-normal subjects, or decreases in signal due to TMS. This filtering of the PubMed search results yielded 6 contrasts and 53 foci from 5 rTMS papers in which $\mathrm{LMI}_{\text {hand }}$ was stimulated (Bestmann et al., 2004; Denslow et al., 2005; Siebner et al., 1999, 2000; Speer et al., 2003). This final set of 5 papers varied by the intensity and frequency of TMS and the data analysis procedures (Table 1). Image acquisition and TMS were simultaneous in three studies (Bestmann et al., 2004; Denslow et al., 2005; Speer et al., 2003). This was not the case for two PET studies (Siebner et al., 1999, 2000), but they were not excluded since TMS was simultaneous with uptake of the tracer. Generally, meta-analytic procedure dictates that ROI analyses be excluded; however, in the case of $\mathrm{LMI}_{\text {hand }}$ TMS studies, this limitation would restrict the total pool of studies to only two (Bestmann et al., 2004; Denslow et al.,
2005). Thus, a heterogeneous mixture of whole-brain analyses (Bestmann et al., 2004; Denslow et al., 2005), ROI analyses (Siebner et al., 1999, 2000), and correlation analyses (Speer et al., 2003) were included in the meta-analysis.

\section{Voluntary movement literature search}

To compare the $\mathrm{LMI}_{\text {hand }}$ TMS meta-analysis network to the network for overt, voluntary hand movement, an ALE metaanalysis of finger tapping studies was performed. PubMed was searched for all studies in which right-handed finger tapping of simple sequences was imaged using fMRI or PET. Again, the pool of studies was restricted to include only those studies that published their results in the form of stereotactic $(x, y, z)$ coordinates. Studies were additionally excluded in the usual fashion if they reported only individual subject data, data from non-normal subjects, deactivations, or ROI analyses. In contrast to the TMS metaanalysis, this last restriction was made possible by the fact that the finger tapping task has frequently been employed in functional neuroimaging, thus yielding an extremely rich body of literature. Contrasts were isolated in which right-handed subjects performed simple tapping of either all of the fingers on the right hand, or the index or ring finger alone, was compared to rest (i.e., Tapping vs. Rest). The literature search and resultant filtering yielded 16 papers, 20 contrasts, and 204 foci (Table 2).

\section{ALE meta-analysis procedure}

ALE was performed in the BrainMap environment (Fox and Lancaster, 2002; Laird et al., 2005b). Upon insertion into the database, foci flagged as belonging to MNI space were automatically converted to Talairach space using the icbm2tal algorithm (Lancaster et al., 2007), which has shown to provide improved fit over the mni2tal transform (Brett, 1999). Each included foci was

Table 2

Input data for ALE meta-analysis of finger tapping studies

\begin{tabular}{lrll}
\hline Author & $n$ & Contrast & Modality \\
\hline Aoki & 10 & Index finger - Rest & PET \\
Aoki & 10 & Ring finger - Rest & PET \\
Aoki & 10 & Double finger tapping - Rest & PET \\
Blinkenberg & 8 & Finger tapping vs. Rest & PET \\
Calautti & 7 & Right-hand tapping vs. Rest, young adults & PET \\
Catalan & 13 & Sequence 16 vs. Rest, controls & PET \\
Denslow & 11 & Volitional movement & fMRI \\
Gelnar & 8 & Motor vs. Rest & fMRI \\
Joliot, 1998 & 5 & Finger tapping - Rest & PET \\
Joliot, 1999 & 8 & Finger tapping, PET & PET \\
Joliot, 1999 & 8 & Finger tapping, fMRI (average) & fMRI \\
Kawashima & 6 & Increased movement, all movements & PET \\
& & compared to rest & \\
Lehericy & 12 & Simple - Rest & fMRI \\
Lehericy & 12 & Scale - Rest & fMRI \\
Lerner & 10 & Tapping - Rest, normals & PET \\
Mattay & 8 & Dominant hand simple motor & fMRI \\
Riecker & 10 & Main effects during index finger movements, & fMRI \\
& & young adults & \\
Rounis & 16 & Main effects of movement & PET \\
Sadato & 9 & Right unimanual vs. Rest & PET \\
Yoo & 10 & Group-level finger tapping activation & fMRI \\
\hline
\end{tabular}

16 papers with 20 contrasts and 204 foci were included in the finger tapping (overt movement) meta-analysis (listed in alphabetical order by first author). $n=$ number of subjects included in a given study. 
blurred with a FWHM of $12 \mathrm{~mm}$. The ALE statistic was computed for every voxel in the brain according to the algorithm developed by Turkeltaub et al. (2002). A permutation test that included 5000 permutations was performed to determine the statistical significance of the ALE results and those results were thresholded at $P<0.05$, FDR-corrected (Laird et al., 2005a). ALE results were output to image format and overlaid onto a Talairach template (Kochunov et al., 2002).

\section{Structural equation modeling}

The locations of the regions of interest (ROIs) selected for inclusion in the structural equation model of TMS/PET data were taken directly from the results of the ALE meta-analysis of the TMS literature. Averaged normalized PET counts were extracted in each ROI from cubic volumes of $125 \mathrm{~mm}^{3}$ in the TMS/PET data. These data were input to Amos 7.0 (Arbuckle, 2006a) for SEM analysis. Each ROI was modeled as an observed variable, while TMS intensity was modeled as a separate observed variable directly modulating the stimulated site (Fox et al., 2006). The values of this intensity variable were 0 (representing the Rest condition), 3 (representing the condition in which TMS was applied at $75 \%$ of the motor threshold (MT)), 4 (representing the 100\% MT condition), and 5 (representing the $125 \%$ MT condition). Error terms on each ROI were modeled as unknown, exogenous variables and the regression weights of these error terms were set to a value of 1 .

\section{Model-generating specification searches}

The model-generating path analysis procedure began with a unidirectional input path (i.e., using an "instrumental variable" strategy as defined in Appendix A) that linked the TMS intensity variable to the left primary motor cortex ( $\mathrm{LMI}_{\text {hand }}$ ) variable (site of stimulation). From this single path, it was hypothesized that stimulation from $\mathrm{LMI}_{\text {hand }}$ would propagate to one or more of the other ROIs. Based on this reasoning, 10 unidirectional output paths were drawn from $\mathrm{LMI}_{\text {hand }}$ to all of the other brain regions. These paths were tested to identify the node or nodes of propagation using model-generating specification searches, allowing for multiple candidate models to be simultaneously tested using optional unidirectional path loadings to determine the most appropriate choice based on fit, parsimony, and interpretability (Arbuckle, 2006b). The path from the intensity variable to $\mathrm{LMI}_{\text {hand }}$ was set as a required path; all other paths originating from $\mathrm{LMI}_{\text {hand }}$ were set as optional. The optional paths were combined in various permutations to form the candidate models, thereby allowing for the assessment of which collection of path tracings produced the model exhibiting the highest degree of validity or goodness relative to the given data.

Two different types of specification searches were utilized: all subsets and stepwise. The preferred type of search was all subsets since this option tests all possible combinations of optional paths. In cases of large numbers of optional paths, a stepwise specification search was chosen to reduce computational time by using a combined forward and backward heuristic search strategy. During a forward specification search, the model with no optional paths is fitted first, then optional paths are added one at a time to determine which path provides the largest reduction in discrepancy between the candidate model and the given data. In a backward specification search, all optional paths are initially tested, then removed one by one. The stepwise search alternates these two search strategies (beginning with a forward search), and records the best one-optional-path model, two-optionalpath model, etc. The search continues by adding or subtracting optional paths until a forward and a backward search is completed with no improvement in model fit (Arbuckle, 2006b).

\section{Model fit statistics}

All specified models of signal output from $\mathrm{LMI}_{\text {hand }}$ were tested and compared. The model with the lowest Bayes information criterion (BIC) and Browne-Cudeck criterion (zero-scale) (BCC) values was selected as the model that represented the optimal model relative to the actual data. The BIC is defined as:

$\mathrm{BIC}=\hat{C}+q \ln \left(N^{(1)} p^{(1)}\right)$,

where $\hat{C}$ is the minimum value of the discrepancy function, $q$ is the number of parameters, $N^{(1)}$ is the number of observations in group 1, and $p^{(1)}$ is the number of observed variables in group 1 . The BCC is defined as:

$\mathrm{BCC}=\hat{C}+2 q \frac{\sum_{g=1}^{G} b^{(g)} \frac{p^{(g)}\left(p^{(g)}+3\right)}{N^{(g)}-p^{(g)}-2}}{\sum_{g=1}^{G} p^{(g)}\left(p^{(g)}+3\right)}$,

where $G$ is the number of groups, $p^{(g)}$ is the number of observed variables in group $g$, and $b^{(g)}=n \frac{N^{(g)}}{N}$, where $N^{(g)}$ equals the number of observations in group $g$, and $N$ equals the total number of observations in all groups combined. The BIC and BCC are predictive fit indices that are used to select among competing models that are fit to the same data, and provide information regarding comparative model fit and model complexity. That is, the BIC and BCC reflect a trade-off between the fit of a model and the effective number of parameters in the model. The BIC and BCC statistics were chosen as the ranking criteria over another possible relative fit measure, the Akaike information criterion (AIC), since the AIC is not based on a strictly Bayesian approach and therefore is not viewed with as much reliability or accuracy as the BCC or BIC (Raftery, 1993; Arbuckle, 2006b). Selection of the model corresponding to the lowest BIC and $\mathrm{BCC}$ values identified the model that contained the next level of nodes in the network following $\mathrm{LMI}_{\text {hand }}$ stimulation. If the lowest $\mathrm{BCC}$ and $\mathrm{BIC}$ values specified different models, greater weight was given to the $\mathrm{BCC}$ criterion since the $\mathrm{BIC}$ imposes a greater penalty for model complexity than does the BIC. This reasoning is a conservative choice that was made when considering the large degree of complexity of the modeled system.

Selection of the model corresponding to the lowest BCC and BIC identified the model that contained the next level of nodes in the network following $\mathrm{LMI}_{\text {hand }}$ stimulation. From those second-level nodes, the next stage of the analysis proceeded and optional paths were drawn to all other regions in the network. This method of signal tracking and model testing to determine the path outputs from all subsequent nodes continued until no further paths were suggested via specification search.

While the BIC and BCC indices are appropriate when comparing candidate models during the model-generating procedure, once the final model was selected, it was necessary to evaluate the goodness of this model using appropriate measures of absolute and relative fit. Assessment of model fit is not a simple process, and there exists no definitive way to assess how well a model represents an actual data matrix (Bollen, 1989). In fact, there is no single statistical test that can be performed in order to identify that a given model is the correct model (Schumacker and Lomax, 2004). Therefore, a large number of descriptive statistics have been developed to aid researchers in evaluating the adequacy or goodness of model relative to a given data (covariance) matrix. In fact, multiple models can simultaneously fit the data equally well. Thus, the final model was evaluated using a number of descriptive fit statistics. To examine overall model adequacy, the 
Table 3

Results of the TMS meta-analysis

\begin{tabular}{|c|c|c|c|c|c|c|}
\hline Region of interest (ROI) & $\mathrm{BA}$ & $x$ & $y$ & $z$ & ALE & $\mathrm{mm}^{3}$ \\
\hline $\begin{array}{l}\text { Left primary sensorimotor } \\
\text { cortex }\left(\mathrm{LMI}_{\text {hand }}\right)\end{array}$ & 4.3 & -30 & -22 & 50 & 0.0100 & 9208 \\
\hline $\begin{array}{l}\text { Left posterior parietal } \\
\text { cortex (LPPC) }\end{array}$ & 5 & -30 & -42 & 62 & 0.0061 & s.c. \\
\hline $\begin{array}{l}\text { Left ventral posterolateral nucleus } \\
\text { of the thalamus (LTHvpl) }\end{array}$ & - & -4 & -26 & 0 & 0.0085 & 3944 \\
\hline $\begin{array}{l}\text { Left ventral lateral nucleus of the } \\
\text { thalamus (LTHvl) }\end{array}$ & - & -11 & -16 & 14 & 0.0075 & s.c. \\
\hline Left ventral premotor area (LPMv) & 6 & -50 & -4 & 18 & 0.0074 & 3576 \\
\hline Cingulate gyrus (Cing) & 24 & 0 & -2 & 44 & 0.0067 & 3024 \\
\hline Supplementary motor area (SMA) & 6 & 0 & -14 & 58 & 0.0065 & s.c. \\
\hline $\begin{array}{l}\text { Right secondary somatosensory } \\
\text { cortex (RSII) }\end{array}$ & 40 & 42 & -28 & 14 & 0.0071 & 2672 \\
\hline $\begin{array}{l}\text { Left secondary somatosensory } \\
\text { cortex (LSII) }\end{array}$ & 40 & -42 & -28 & 16 & 0.0091 & 2552 \\
\hline $\begin{array}{l}\text { Right ventrolateral } \\
\text { thalamus (RTHvl) }\end{array}$ & - & 8 & -14 & 14 & 0.0074 & 1104 \\
\hline Right cerebellum (RCer) & - & 10 & -46 & -10 & 0.0059 & 800 \\
\hline
\end{tabular}

11 ROIs were returned by the ALE meta-analysis of published studies that investigated TMS of left primary motor cortex in normal subjects.

s.c. $=$ same cluster as listed on previous line; $\mathrm{ALE}=$ maximum activation likelihood estimation value for the given cluster; $\mathrm{mm}^{3}=$ cluster volume.

likelihood ratio chi-square $\left(\chi^{2}\right)$ statistic was used to test whether the discrepancy between the implied versus actual covariance matrix was for statistically different (Price et al., 2002). Theoretical models that fit the given data perfectly have a $\chi^{2}$ value of zero. Thus, it is desirable to achieve a non-significant $\chi^{2}$ value, since a high $P$ value indicates that the tested model cannot be refuted. Another global fit measure was computed, known as the root mean square error of approximation (RMSEA) and its associated 90th percentile confidence interval $(90 \%$ $\mathrm{CI}_{\text {RMSEA }}$ ). Obtaining an RMSEA value of less than 0.05 indicates a good model fit. Lastly, the comparative fit index (CFI) and TuckerLewis index (TLI) (relative fit indices) were computed, both of which range from values of 0 (no fit) to 1 (perfect fit), with values of 0.95 or greater indicating a good model fit (Schumacker and Lomax, 2004).

\section{Results}

\section{ALE meta-analysis}

ALE meta-analysis of the TMS literature yielded 11 ROIs: the hand region of the left primary sensorimotor cortex $\left(\mathrm{LMI}_{\text {hand }}\right)$, left posterior parietal cortex (LPPC), left ventral premotor cortex (LPMv), left and right secondary somatosensory cortex (LSII and RSII), left and right ventral lateral nucleus of the thalamus (LTHvl and RTHvl), left ventral posterolateral nucleus of the thalamus (LTHvpl), bilateral supplementary motor area (SMA), bilateral cingulate (Cing), and right cerebellum (RCer) (Table 3 ). To confirm that the TMS meta-analysis yielded reasonable results, the TMS ALE maps were compared to two images: (1) the activation map for the $125 \% \mathrm{MT}$ - Rest contrast in the TMS/PET data and (2) the ALE results of the overt movement (finger tapping) meta-analysis. Fig. 1 shows the agreement between the TMS meta-analysis of the literature and the suprathreshold activation results in the experimental TMS data. Overlap of the TMS metaanalysis and activation in the 7 TMS subjects was observed in 8 of

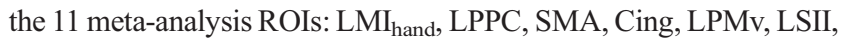
LTHvpl, and RCer. No overlap was observed between bilateral thalamus (RTHvl and LTHvl) and right secondary somatosensory cortex (RSII). Fig. 2 shows the agreement between the TMS and finger tapping meta-analyses (Table 4). Overlap between the two metaanalyses was seen in 8 of the 11 TMS ROIs: $\mathrm{LMI}_{\text {hand }}$ LPPC, SMA, Cing, LPMv, LSII, LTHvl, and RCer. No overlap was observed in the right ventral lateral nucleus of the thalamus (RTHvl), right secondary somatosensory cortex (RSII), and left ventral posterolateral nucleus of the thalamus (LTHvpl). We judge these overlap data as reasonable confirmation of the ROI selection method presented here.

\section{Structural equation modeling}

The sample size of the TMS/PET data was 56 (7 subjects $\times 4$ conditions $\times 2$ trials) with 23 variables: 11 observed, endogenous variables for each ROI; 11 unobserved, exogenous variables representing the error terms loaded onto those ROIs; and 1 observed, exogenous variable for TMS intensity. Given the required path of intensity loaded upon $\mathrm{LMI}_{\text {hand }}$, the possible loadings from $\mathrm{LMI}_{\text {hand }}$ to all other nodes in the network (10 optional paths) were investigated. Specification search (all subsets) revealed that from the $\mathrm{LMI}_{\text {hand }}$ stimulation site, activation propagated to five regions: supplementary motor area (SMA), cingulate gyrus (Cing), left ventral lateral nucleus of the thalamus (LTHvl), right secondary somatosensory cortex (RSII), and right cerebellum (RCer). We termed these loadings "first-level paths" or the "first-level cascade" to indicate that they were the first nodes to receive input from $\mathrm{LMI}_{\text {hand }}$ following stimulation; they can be viewed in Fig. 3a (red paths).

To determine the second level of paths, optional loadings were added from each of the five first-level ROIs to all other nodes available ( 5 ROIs $\times 10$ possible nodes $=50$ optional loadings $)$. The five previous paths from $\mathrm{LMI}_{\text {hand }}$ were relabeled as required, not optional.

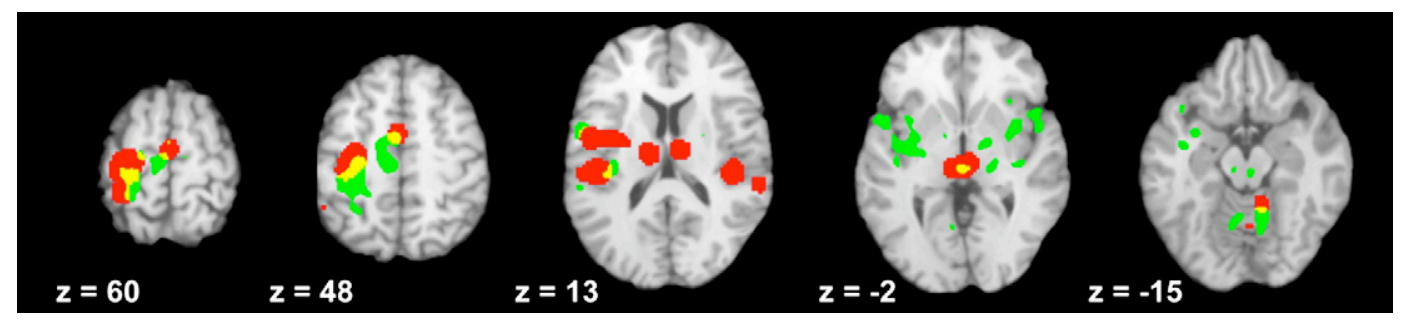

Fig. 1. Overlap between TMS meta-analysis and activation results of the TMS/PET data. These axial slices show the overall agreement between the results of the ALE meta-analysis of 7 TMS studies $(P<0.05)$ (red) and the activation data from 7 subjects acquired at the Research Imaging Center in the contrast of $125 \%$ MT TMS-Rest $(P<0.05)$ (green). Areas of overlap between these two maps (yellow) were found in the left primary motor cortex $\left(\mathrm{LMI}_{\text {hand }}\right)$, left posterior parietal cortex (LPPC), supplementary motor area (SMA), cingulate gyrus (Cing), left ventral premotor area (LPMv), left secondary somatosensory cortex (LSII), left ventral posterolateral nucleus of the thalamus (LTHvpl), and right cerebellum (RCer). 


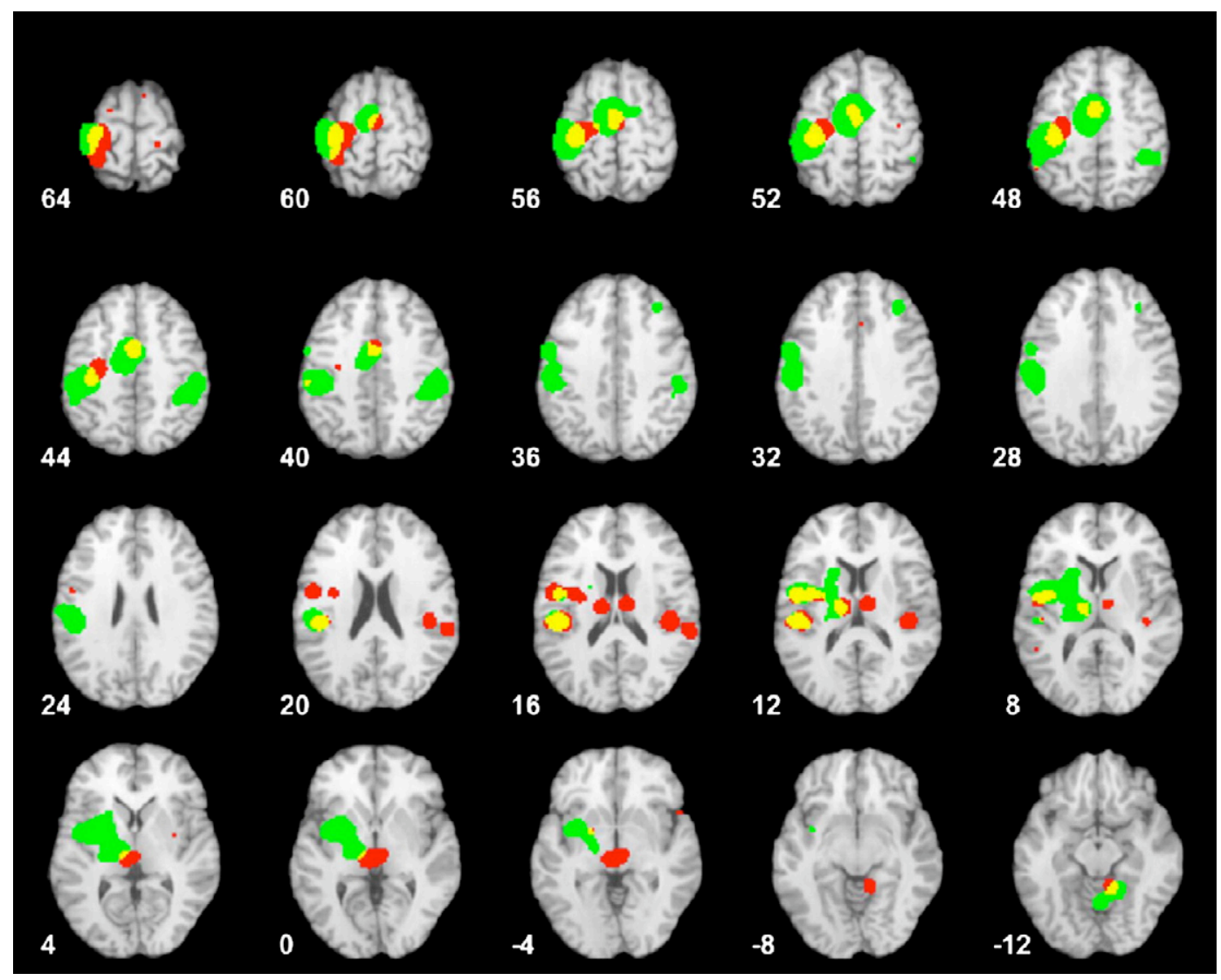

Fig. 2. Overlap between TMS meta-analysis and finger tapping meta-analysis. Shown here are the results of the ALE meta-analysis of 7 TMS studies $(P<0.05)$ (red), the results of the ALE meta-analysis of 16 finger tapping studies $(P<0.05)$ (green) and the overlap between them (yellow). Overlap between these two ALE maps exists in the left motor cortex ( $\left.\mathrm{LMI}_{\text {hand }}\right)$, left posterior parietal cortex (LPPC), supplementary motor area (SMA), cingulate gyrus (Cing), left ventral premotor area (LPMv), left secondary somatosensory cortex (LSII), left ventral lateral nucleus of the thalamus (LTHvl), and right cerebellum (RCer).

Due to the large number of possible paths in this level of the analysis, a stepwise specification search was performed, which returned a best fit model with 17 additional loadings as seen in Fig. $3 b$ (green paths). There were 9 ROIs that received input due to the second-level paths: LMI $_{\text {hand }}$, LSII, LTHvl, Cing, RTHvl, RSII, LPPC, LTHvpl, and LPMv (only the SMA and RCer received no input at the secondary level).

To determine the third-level paths, optional loadings were drawn for the 9 nodes listed above; both first-level and secondary-level paths were set as required. Stepwise specification search revealed only five additional loadings in the model: RTHvl to LPMv, LPMv to LTHvpl

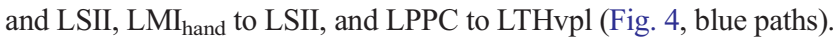
A fourth-level specification search with optional loadings originating from these three additional nodes (LPMv, LTHvpl, and LSII) revealed no other paths should be added to the model.

\section{Model fit statistics}

Measures of model goodness and fit for all three levels of specification searches (Table 5) show that while the initial model of only 6 loadings did not fit the data well, additional levels of analysis improved the fit of the model dramatically. Specifically, the last round of specification searches generated an extremely large increase in the $P$ value, resulting in the non-significance of the model $(P=0.004$ for second-level paths to $P=0.981$ for thirdlevel paths; Table 5). This large transition possibly indicates the introduction of critical paths during that stage of the analysis. After the last round of specification searches, the estimates were cal- culated and it was determined that the overall final model fit was outstanding $\left(\chi^{2}=22.150, d f=38, P=0.981, \mathrm{CFI}=1.0, \mathrm{TLI}=1.0\right.$, $\left.\mathrm{RMSEA}=0.000,90 \% \mathrm{CI}_{\mathrm{RMSEA}}=0.000-0.000\right)$. These statistics

Table 4

Results of the finger tapping meta-analysis

\begin{tabular}{|c|c|c|c|c|c|c|}
\hline Region of interest (ROI) & BA & $x$ & $y$ & $z$ & ALE & $\mathrm{mm}^{3}$ \\
\hline $\begin{array}{l}\text { Left primary sensorimotor } \\
\text { cortex }\left(\mathrm{LMI}_{\text {hand }}\right)\end{array}$ & 4.3 & -38 & -28 & 52 & 0.0345 & 25,288 \\
\hline $\begin{array}{l}\text { Left secondary somatosensory } \\
\text { cortex (LSII) }\end{array}$ & 40 & -48 & -26 & 22 & 0.0145 & s.c. \\
\hline $\begin{array}{l}\text { Left ventral premotor } \\
\text { area (LPMv) }\end{array}$ & 6 & -34 & -4 & 4 & 0.0178 & 15,504 \\
\hline $\begin{array}{l}\text { Left ventral lateral nucleus of } \\
\text { the thalamus (LTHvl) }\end{array}$ & - & -14 & -18 & 6 & 0.0169 & s.c. \\
\hline $\begin{array}{l}\text { Supplementary motor } \\
\text { area (SMA) }\end{array}$ & 6 & -6 & -10 & 50 & 0.0326 & 13,104 \\
\hline Right cerebellum (RCer) & - & 18 & -50 & -20 & 0.0216 & 6544 \\
\hline $\begin{array}{l}\text { Right primary motor } \\
\text { cortex }\left(\mathrm{RMI}_{\text {hand }}\right)\end{array}$ & 4.3 & 50 & -28 & 42 & 0.0106 & 4872 \\
\hline Left cerebellum (LCer) & - & -22 & -56 & -24 & 0.0101 & 1072 \\
\hline Right middle frontal & 9 & 34 & 32 & 32 & 0.0088 & 656 \\
\hline
\end{tabular}
gyrus (RMiFG)

9 ROIs were returned by the ALE meta-analysis of published studies that investigated simple finger tapping of the dominant hand by right-handed, normal subjects. 

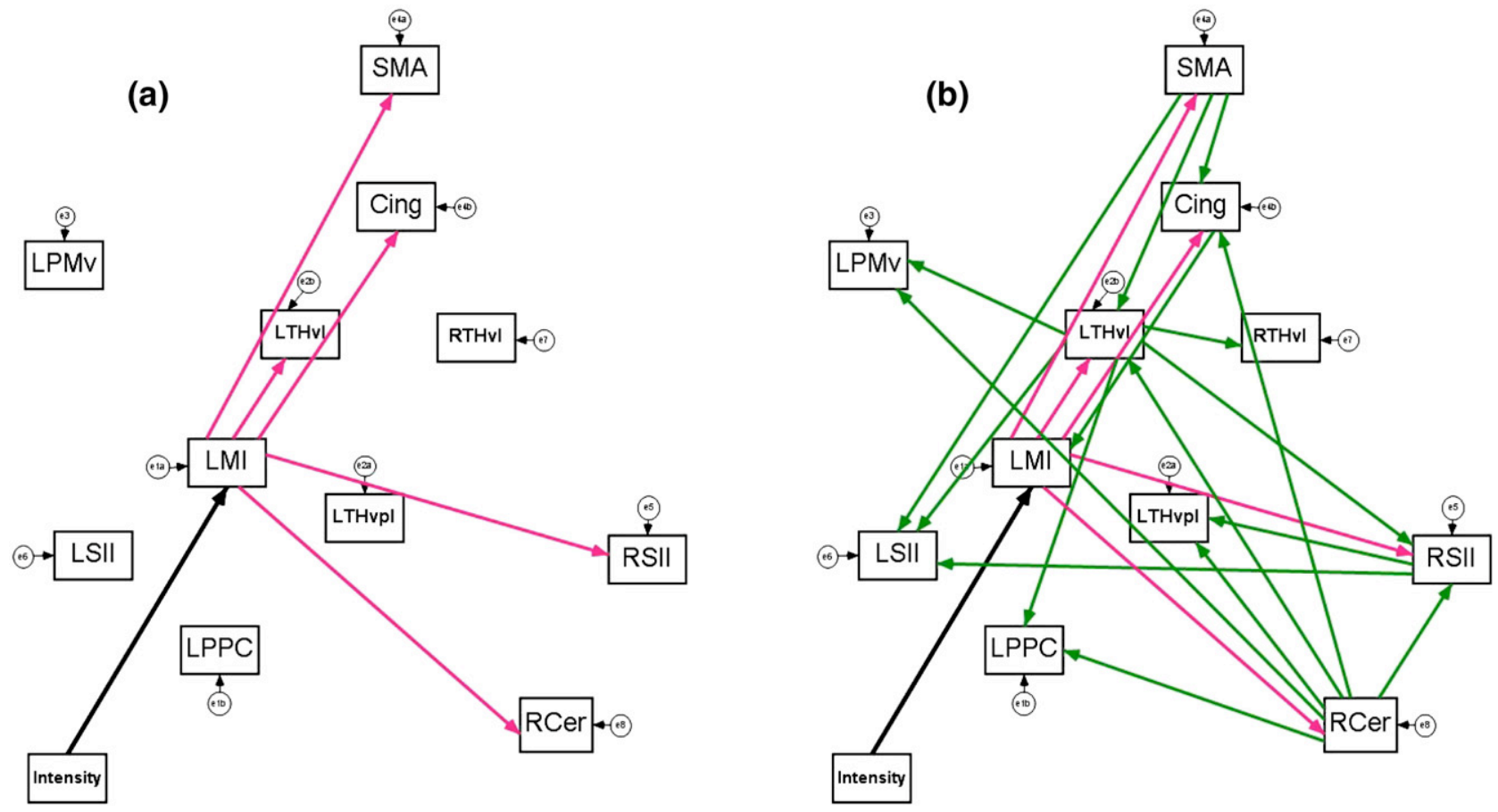

Fig. 3. First-level and second-level paths in the LMI TMS model. (a) Following stimulation with TMS, exploratory SEM revealed that the signal propagated from $\mathrm{LMI}_{\text {hand }}$ to five nodes, designated by the five red paths to supplementary motor area (SMA), cingulate gyrus (Cing), left ventral lateral nucleus of the thalamus (LTHvl), right secondary somatosensory cortex (RSII), and right cerebellum. We termed these "first-level paths." (b) Propagation of the TMS signal from those five nodes to all other nodes was determined next, generating the second-level paths in the model (green paths). Error terms are represented by the circular variables.

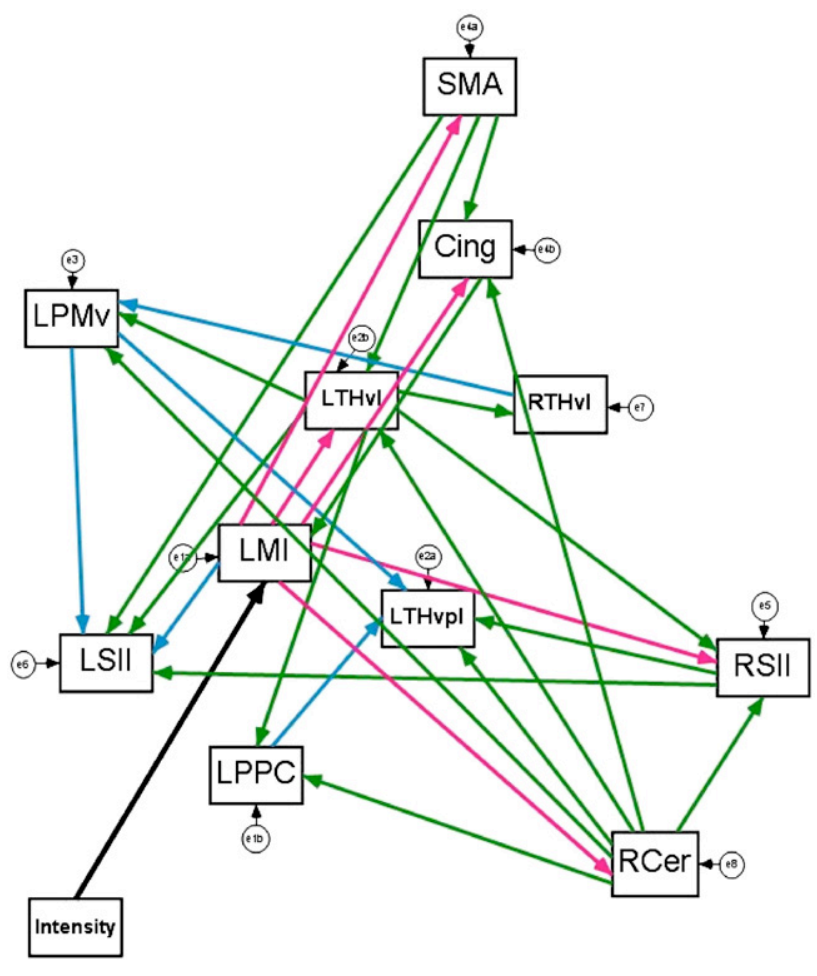

Fig. 4. Final model of $\mathrm{LMI}_{\text {hand }}$ connectivity. Exploratory analysis of $11 \mathrm{TMS}$ ROIs using SEM revealed a complex network of $\mathrm{LMI}_{\text {hand }}$ connectivity that fit the data extremely well $\left(\chi^{2}=22.150, d f=38, P=0.981, \mathrm{CFI}=1.0, \mathrm{TLI}=1.0\right.$, RMSEA $\left.=0.000,90 \% \mathrm{CI}_{\text {RMSEA }}=0.000-0.000\right)$. Red paths $=$ first-level paths from LM1; green paths $=$ second-level paths; blue paths $=$ third-level paths . Error terms are represented by the circular variables. indicate little difference between the sample variance-covariance matrix and the reproduced variance-covariance matrix implied by this model (Schumacker and Lomax, 2004). Regression weights for these paths can be seen in Table 6. A non-recursive (i.e., interdependent systems) loop is present in the final model, involving the nodes of left motor cortex ( $\mathrm{LMI}_{\text {hand }}$ ), right cerebellum (RCer), supplementary motor area (SMA), and cingulate gyrus (Cing). Non-recursive relations pose a unique challenge during simulta-

Table 5

Fit statistics of the models in each level of analysis

\begin{tabular}{lrrllll}
\hline & $C$ & \multicolumn{1}{c}{$C-d f$} & $\mathrm{BCC}(0)$ & $\mathrm{BIC}(0)$ & $C / d f$ & $P$ \\
\hline First-level paths & 410.998 & 350.998 & 0.000 & 1.090 & 6.850 & 0.000 \\
Second-level paths & 71.232 & 28.232 & 0.000 & 1.533 & 1.657 & 0.004 \\
Third-level paths & 22.150 & -15.850 & 0.000 & 0.000 & 0.583 & 0.981 \\
\hline
\end{tabular}

Overall model goodness improved as more paths were added via specification search. The first-level cascade contained only the intensity to $\mathrm{LMI}_{\text {hand }}$ loading and the 5 loadings originating from $\mathrm{LMI}_{\text {hand }}$ ( 6 total paths; Fig. 3a, red paths). The second-level paths added 17 additional loadings to the model (23 total paths; Fig. 3b, green paths). Lastly, 5 loadings were added in the third-level of specification searches (28 total paths; Fig. 4, blue paths).

$C=$ minimum value of the discrepancy $\left(\chi^{2}\right.$ value $) ; d f=$ degrees of freedom; $\mathrm{BCC}(0)=$ Browne-Cudeck criterion, scaled such that the lowest $\mathrm{BCC}$ is zero, with all other values being positive; $\operatorname{BIC}(0)=$ Bayes information criterion, also scaled such that the minimum value for the tested models equals zero; $P=$ probability of getting as large a discrepancy as occurred with the present sample ( $P$ value for testing that the model fits perfectly in the given population). For both $\mathrm{BCC}$ and BIC, smaller values indicate a higher degree of goodness than larger values. For $P$, larger values indicate a better fit of the model. 
Table 6

Maximum likelihood estimates of the final model

\begin{tabular}{|c|c|c|c|c|c|}
\hline Path & Estimate & Std. Estimate & S.E. & CR & $P$ \\
\hline LMI $\leftarrow$ Intensity & 36.228 & 0.562 & 19.921 & 1.819 & 0.069 \\
\hline LTHvl $\leftarrow$ LMI & -0.419 & -0.192 & 0.234 & -1.792 & 0.073 \\
\hline LTHvl $\leftarrow$ SMA & 1.336 & 0.416 & 0.333 & 4.013 & $* * *$ \\
\hline LTHvl $\leftarrow$ RCer & 1.079 & 0.536 & 0.210 & 5.151 & $* * *$ \\
\hline RTHvl $\leftarrow$ LTHvl & 0.766 & 0.921 & 0.044 & 17.564 & $* * *$ \\
\hline $\mathrm{RSII} \leftarrow \mathrm{LMI}$ & 0.550 & 0.500 & 0.108 & 5.112 & $* * *$ \\
\hline $\mathrm{RSII} \leftarrow \mathrm{LTHvl}$ & 0.177 & 0.351 & 0.056 & 3.167 & 0.002 \\
\hline $\mathrm{RSII} \leftarrow \mathrm{RCer}$ & 0.439 & 0.432 & 0.115 & 3.831 & $* * *$ \\
\hline $\mathrm{LPPC} \leftarrow \mathrm{RCer}$ & 0.426 & 0.481 & 0.114 & 3.727 & $* * *$ \\
\hline LPPC $\leftarrow$ LTHvl & -0.302 & -0.687 & 0.057 & -5.340 & $* * *$ \\
\hline $\mathrm{LPMv} \leftarrow \mathrm{RTHvl}$ & 0.355 & 1.000 & 0.084 & 4.227 & $* * *$ \\
\hline $\mathrm{LPMv} \leftarrow \mathrm{LTHvl}$ & -0.215 & -0.738 & 0.075 & -2.870 & 0.00 \\
\hline $\mathrm{LPMv} \leftarrow \mathrm{RCer}$ & 0.289 & 0.493 & 0.065 & 4.439 & $* * *$ \\
\hline LTHvpl $\leftarrow$ RSII & 0.369 & 0.296 & 0.114 & 3.234 & 0.001 \\
\hline LSII $\leftarrow$ RSII & 1.289 & 0.624 & 0.185 & 6.975 & $* * *$ \\
\hline LSII $\leftarrow$ LMI & -0.505 & -0.222 & 0.197 & -2.567 & 0.010 \\
\hline $\mathrm{LSII} \leftarrow \mathrm{LPMv}$ & 0.787 & 0.220 & 0.296 & 2.662 & 0.008 \\
\hline LTHvpl $\leftarrow$ LPPC & 0.378 & 0.265 & 0.117 & 3.236 & 0.001 \\
\hline LTHvpl $\leftarrow$ RCer & 0.312 & 0.246 & 0.131 & 2.373 & 0.018 \\
\hline LTHvpl $\leftarrow$ LPMv & 0.925 & 0.429 & 0.208 & 4.450 & $* * *$ \\
\hline $\mathrm{LSII} \leftarrow \mathrm{SMA}$ & -0.686 & -0.205 & 0.264 & -2.594 & 0.009 \\
\hline LSII $\leftarrow$ LTHvl & 0.221 & 0.212 & 0.105 & 2.097 & 0.036 \\
\hline Cing $\leftarrow$ SMA & 0.379 & 0.429 & 0.143 & 2.659 & 0.008 \\
\hline LMI $\leftarrow$ Cing & -1.578 & -0.946 & 1.340 & -1.178 & 0.239 \\
\hline Cing $\leftarrow \mathrm{LMI}$ & 0.460 & 0.767 & 0.144 & 3.186 & 0.001 \\
\hline $\mathrm{SMA} \leftarrow \mathrm{LMI}$ & 0.319 & 0.469 & 0.125 & 2.550 & 0.011 \\
\hline RCer $\leftarrow$ LMI & 0.010 & 0.009 & 0.247 & 0.042 & 0.967 \\
\hline Cing $\leftarrow$ RCer & 0.322 & 0.581 & 0.103 & 3.113 & 0.002 \\
\hline
\end{tabular}

After the model-generating procedure SEM, the estimates for the final model were calculated as seen in Fig. 4.

Estimate $=$ estimate of the regression weight (e.g., when intensity goes up by 1, $\mathrm{LMI}_{\text {hand }}$ goes up by 36.228); Std. Estimate=estimate of the standardized regression weight (e.g., when intensity goes up by 1 standard deviation, $\mathrm{LMI}_{\text {hand }}$ goes up by 0.562 standard deviations); S.E. $=$ standard error of the regression weight; $\mathrm{CR}=$ critical ratio for regression weight, which is computed by dividing the regression weight estimate by the estimate of its standard error (e.g., for the path from intensity to $\mathrm{LMI}_{\text {hand }}$, the regression weight estimate is 1.819 standard errors above zero); $P=$ level of significance for regression weight; $* * * P<0.001$ (Arbuckle, 2006b).

neous estimation of the model structural coefficients due to an infinite sequence of linear dependencies among nodes. For some regression weights, these dependencies will converge to a set of well-defined relations, thereby exhibiting system stability. To ensure that an adequate level of stability for the network system was achieved (i.e., within the range of -1.0 to +1.0 ; Bentler and Freeman, 1983; Fox, 1980), we used an instrumental variable design strategy (Heise, 1975). Once implemented, the stability of the recursive loops and the entire system was estimated using the full information maximum likelihood (FIML) estimation algorithm (to allow for a sparse variance-covariance matrix) with the ridge regression option invoked. The statistical basis for evaluating the stability of the non-recursive path model is provided by Bentler and Freeman (1983) and is described in Appendix B. The stability index was observed as +0.78 , indicating a mathematically stable solution.

In Table 5, all three values of the Brown-Cudeck criterion (BCC) are zero. Since Amos only uses BCC values in a comparative sense, zero-scaled $\mathrm{BCC}$ values are used in which the lowest $\mathrm{BCC}$ is set to 0 , and the other values are scaled accordingly. Thus, the $\mathrm{BCC}$ values of zero in Table 5 indicate that the three models listed were the lowest of their respective stage of the analysis. The BIC values for the firstand second-level analyses are non-zero, indicating that these were not the models specified by the lowest BIC values. In the two cases of conflict between the BCC and BIC, the BIC specified models with two fewer paths (due to its increased penalty for model complexity), thus returning alternate models that were extremely similar to those generated when using the $\mathrm{BCC}$ as a selection criterion. In the last stage of analysis (third-level paths), the BCC and BIC returned the same model.

\section{Discussion}

The main aim of this study was to use TMS/PET as a novel approach to quantifying effective connectivity during stimulation of the $\mathrm{LMI}_{\text {hand }}$ region. ALE meta-analysis of the TMS literature was used to identify regions of interest (ROIs) that were subsequently analyzed in a TMS/PET data set of 7 subjects using a modelgenerating SEM procedure. Eleven ROIs and TMS intensity were modeled as observed variables in the path model. Exploratory SEM analysis revealed that after TMS of $\mathrm{LMI}_{\text {hand }}$, activation propagated to five regions: the supplementary motor area (SMA), cingulate gyrus (Cing), left ventral lateral nucleus of the thalamus (LTHvl), right secondary somatosensory cortex (RSII), and right cerebellum (RCer). Further signal propagation was determined, resulting in the model observed in Fig. 4. This final model fit the data extremely well, indicating little difference between the actual and modelimplied covariance matrices $\left(\chi^{2}=22.150, d f=38, P=0.981\right.$, $\mathrm{CFI}=1.0$, TLI $=1.0$, RMSEA $=0.000,90 \% \mathrm{CI}_{\mathrm{RMSEA}}=0.000-$ $0.000)$. The validity of this method was established through both quantitative and qualitative measures. Quantitatively, an outstanding fit was observed, and a comparison of fit statistics in previous SEM studies suggested that the exploratory nature of this analysis is responsible for the fit of the model. Qualitatively, the utility of the ROI selection method (via meta-analysis) presented here was confirmed by a comparison with both an analysis of the TMS/PET data and a meta-analysis of overt movement. Consistent findings between the final model presented here and SEM motor studies in humans and motor connectivity studies in the macaque literature were observed. In addition, various features of the final path model were explored, including connections to secondary somatosensory cortex and model criticisms.

\section{Fit statistics of the final path model}

As stated previously, the final model seen in Fig. 4 fit the experimental data extraordinarily well. There are three plausible reasons that might explain why such a good fit was obtained. First, the parametric manner in which TMS was utilized over a wide range of intensities possibly contributed to a greater degree in which the system was probed, leading to the robustness of the results. Second, it is conceivable that the task-independent nature of stimulation with TMS is more conducive in general to the analysis of network interactions. Finally, the exploratory nature of this analysis might have been a critical factor in the strength of the overall results. A brief inspection of the SEM literature in neuroimaging isolated a sub-sample of studies in which the global fit measures of a single model or models were published, rather than the more prevalent $\chi^{2}$ difference values between two models. These studies presented SEM analyses that were either exploratory (Zhuang et al., 2005; Peltier et al., 2007) or confirmatory (Goncalves et al., 2001; Honey et al., 2002; Glabus 
et al., 2003; Erickson et al., 2005; Au Duong et al., 2005; de Marco et al., 2006), and examined a mixture of paradigms and subject groups. After comparing the reported model fit indices and experimental design factors for each paper, the first two possible explanations of the excellent degree of the results presented above were refuted. However, no hypothesis-driven models in the examined studies obtained fit statistics as good as those observed in the exploratory analyses. A reasonable interpretation is then that taking a data-driven approach to generate an optimal model is a more precise way to investigate effective connectivity. While this may seem a trivial conclusion, it is still a point that must be made, given the overwhelming preference in favor of testing a priori SEM models in neuroimaging data. More research is needed to determine if this is an accurate assessment of the data and the analysis method.

\section{Plausibility of $\mathrm{LMI}_{\text {hand }}$ regions}

Overall, excellent agreement was found between the TMS metaanalysis results and (1) the activation map for the $125 \% \mathrm{MT}$ - Rest contrast in the TMS/PET data of 7 subjects, and (2) the meta-analysis results of voluntary right-finger tapping. In comparison to the $125 \%$ MT - Rest activation map, overlap was found in the $\mathrm{LMI}_{\text {hand }}$, LPPC, SMA, Cing, LPMv, LSII, LTHvpl, and RCer (Fig. 1). While the ROIs for SEM analysis could have been selected from the activation map alone, the meta-analysis approach provided a more defensible means of isolating nodes common to a larger group of subjects, thereby reducing variability due to imaging and analysis methods. In general, ROI selection is highly variable among SEM studies. Using metaanalysis as a technique for ROI selection provides a more standardized, less user-dependent strategy. Overlap between the metaanalysis results and the activation results indicates that the present TMS/PET data agree well with other reports in the literature, and gives greater confidence in the selection of motor regions.

Second, in the comparison between the TMS and finger tapping meta-analyses, overlap was seen in the LMI, LPPC, SMA, Cing, LPMv, LSII, LTHvl, and RCer (Fig. 2). Regions present in the TMS meta-analysis that were not observed in the finger tapping metaanalysis are arguably more strongly related to the somatosensory system (LTHvpl), and their omission can be attributed to laterality issues associated with right-handed finger tapping (RTHvl and RSII). Thus, with a few exceptions, the TMS network identified via metaanalysis agrees well with the network of voluntary motor function. These results confirm that the meta-analysis method of ROI selection was successful both in identifying the nodes stimulated with TMS and that these nodes are representative of general motor function.

\section{Plausibility of $L M I_{\text {hand }}$ connections}

The final model generated through exploratory path modeling includes a large number of both ROIs and loadings, which implies a level of complexity appropriate for a neural systems model. The presence of many of the observed paths in the final model have been confirmed in previous SEM studies of self-initiated and externally cued finger movements (Taniwaki et al., 2006) and visually guided motor grasping (Grafton et al., 1994), including various paths connecting left primary motor cortex (LMI), supplementary motor area (SMA), left ventral lateral nucleus of the thalamus (LTHvl), left ventral premotor cortex (LPMv), left ventral posterolateral nucleus of the thalamus (LTHvpl), and right cerebellum (RCer).

In addition to finding agreement between the final model presented here and other published SEM results of human neuroima- ging data, agreement was also found between these results and the macaque literature. The connections in the final model were compared to the connectivity map of the $\mathrm{FI}_{\text {arm }}$ region (the homologue of $\mathrm{MI}_{\text {hand }}$ ) (Karol and Pandya, 1971; Schmahmann and Pandya, 2006). In the present model, direct $\mathrm{LMI}_{\text {hand }}$ connections were observed to the cingulate cortex, supplementary motor area, secondary somatosensory cortex (SII), thalamus, and cerebellum. Convergence exists between these connections and the connectivity of FI in the macaque (Schmahmann and Pandya, 2006), giving further support to the validity of these results. The complexity of the presented model of LMI connectivity is well supported by the macaque literature and likely provides a realistic model of motor system connectivity.

\section{$L M I_{\text {hand }}$ path model features}

In the present model, five regions are directly modulated by $\mathrm{LMI}_{\text {hand }}$ following TMS: SMA, Cing, LTHvl, RSII, and RCer(Fig. 3a, red paths). Thus, there were five nodes that did not receive immediate input from $\mathrm{LMI}_{\text {hand }}$ and were thus not introduced into the model until later in the analysis. For example, left premotor cortex (LPMv) was introduced only after receiving input from the left ventral lateral nucleus of the thalamus (LTHvl) and right cerebellum (RCer). In addition, the left ventral posterolateral nucleus of the thalamus (LTHvpl) was introduced after receiving input from right secondary somatosensory cortex (RSII) and right cerebellum (RCer) (Fig. 3b, green paths). It is interesting to note that all possible nodes were introduced into the model during either the first- or second-level analyses, leaving no new nodes to be introduced in the third-level analysis.

At this point, it is unclear what functional significance can be attributed to the first-, second-, and third-level cascades. The hierarchical nature of the present method adds paths to the model in an iterative fashion, and this model progression is data-driven and guided by the search for the model yielding the best indices of model goodness and fit. It can be seen that the order in which some of these paths were added was merely based on the fact that a region cannot directly load onto another region until the first region receives a loading itself (e.g., LPPC $\rightarrow$ LTHvpl is not added to the model until after the incoming paths to the LPPC are considered). However, one loading (LMI $\rightarrow$ LSII) does not show up until the third-level paths are tested, despite the fact that this particular path was tested in the first-level cascade following $\mathrm{LMI}_{\text {hand }}$ stimulation. It is unknown if the level of analysis ascribed to a loading is a function of the distance traveled from the site of stimulation, the strength of the connection, or the loading's relative influence in the entire network. For now, this issue remains open for exploration in future studies.

The loading of $\mathrm{LMI}_{\text {hand }}$ onto RCer was small (standardized path coefficient $=0.009$ ), but this path had a critical effect on the model as seen in the large number of second-level outgoing paths from cerebellum to RSII, Cing, LTHvl, LTHvpl, LPMv, and LPPC. Investigation of the total effects that each variable had on all the other variables in the model (McIntosh and Gonzales-Lima, 1994) revealed that the average total effects of the right cerebellum on all other variables was the largest in the entire model (average total effects= 0.308). Thus, in this model of $\mathrm{LMI}_{\text {hand }}$ connectivity, it is evident that the right cerebellum is a major relay station for information from $\mathrm{LMI}_{\text {hand }}$ to cortical and subcortical nodes in the network. This finding of the importance of the $\mathrm{LMI}_{\text {hand }}$ to RCer connection, despite its small path coefficient, casts doubt on the practice of filtering out paths with small regression weights. 
Table 6 shows the standardized path coefficients for the loadings in the final model (Fig. 4). Note that several of these coefficients are not significant. Once the model-generating analysis was complete and no more additional loadings were suggested, the lower or insignificant regression weights (e.g., LMI to RCer) were not additionally filtered. To do so would have rendered the exploratory signal tracking procedure unsound. That is, since the goal was to track the TMS signal, once a connection was created it was necessary for future specification searches (e.g., the LMI to RCer loading was introduced during the first-level cascade and therefore was relevant to all five outgoing paths from the RCer determined in the second-level model).

In particular, Table 6 shows that the path from Intensity to $\mathrm{LMI}_{\text {hand }}$ is not significant $(P=0.069)$, yet it is clear that the data supports the inclusion of this path. To illustrate this, in the final model we labeled the Intensity $\rightarrow$ LMI connection as optional and tested the two candidate models. The model that included the connection obtained much lower BCC and BIC values $(0.000$ for both criteria) as compared to the model that omitted this path $(\mathrm{BCC}=$ 4.646 and $\mathrm{BIC}=3.239$ ). In terms of the absolute and relative fit statistics, excluding this path resulted in a decrease in the fit of the data to the given model $(P<0.981$ with the path, as compared to $P<0.867$ without the path). Thus the data does support this path.

The regression weights (unstandardized $=36.22$, standardized $=$ 0.562) were observed as being not statistically significant, but they are classified as being "large" (Cohen, 1988). Mathematically, the test of statistical significance is equal to the product of the effect size and the size of the study (sample size). Although the regression of LMI on Intensity was not statistically significant, the magnitude of the regression weight is practically important. For correlations, the effect size is the coefficient of determination $\left(R^{2}\right)$ and indicates the variability in the model that can be predicted from the relationship between the two variables. The importance of the effect size in this case is evidenced by the regression path exhibiting a coefficient of determination of 0.3158 . That is, $32 \%$ of the variability in the overall model was accounted for or explained by this single path. From a practical perspective, this effect is reasonable, considering the large physiological effect delivered to the site of stimulation (i.e., LMI) by the TMS coil. Thus, while the regression weights were found to be not statistically significant, this is a result of the small sample size, and not due to any weakness in the final model.

\section{Path connections to secondary somatosensory cortex (SII)}

In the final TMS connectivity model (Fig. 4), $\mathrm{LMI}_{\text {hand }}$ is found to act directly upon both left and right secondary somatosensory cortex (SII). However, these two loadings are very different. LMI to RSII was added as a first-level path, right after LMI received input from TMS. During this stage of the analysis, the data did not support a connection from LMI to LSII. As mentioned previously, this path was not added until the third-level analysis. Between the first-level and third-level analyses, only one variable acted upon LMI: the cingulate gyrus. Thus, the LMI to LSII connection was only made possible after LMI received input from the cingulate cortex. This was also accompanied by a loading of RSII onto LSII. We hypothesize that the nodes in these interactions (LMI, RSII, LSII, and Cing) are particularly important in the $\mathrm{LMI}_{\text {hand }}$ connectivity network. The LMI $\rightarrow$ Cing $\rightarrow$ LMI nonrecursive loop seems especially significant since the cingulate gyrus was found to have a direct effect only on LMI, and conversely, the only node to directly input LMI was the cingulate gyrus. Our interpretation of these observations is that the LMI/Cing feedback loop is of critical influence in the present network.

\section{Model criticisms and limitations}

There are three major criticisms of the final model. The first criticism is that this model does not include a variable for primary somatosensory cortex (SI). The aim here was to remain very strict in only extracting data from ROIs explicitly returned by the metaanalysis. The largest ALE cluster returned centered on left primary motor cortex (LMI), which was connected to a secondary cluster in BA 5 in posterior parietal cortex (LPPC). The results did reveal that activation of left SI was included in this large frontal-parietal cluster, contained in the zone bridging the primary motor cortex to PPC. However, since SI was not represented as a well-defined sub-cluster in the ALE results, it was not possible to include this region as a node in the path model. Hence, the coupled interaction between MI and SI was not dissociated using the current methodology.

The second major criticism of the model is that a second-level path was found from the left to the right ventral lateral nucleus of the thalamus, despite the fact that there is no known connection between these nuclei (Carpenter, 1985). There are two possible reasons why this path was generated: (1) these regions are located very close to each other in space, resulting in the acquisition of highly correlated data that is not representative of functionally connected regions; or (2) the LTHvl to RTHvl connection in the model is an indirect one, possibly modulated by an unknown variable not included in the existing network. Analysis of the total effects of each variable in the model initially suggested the former cause. That is, the effects of all other variables on both the LTHvl and RTHvl were nearly identical to each other, indicating that the two nodes received input more as a single entity rather than two distinct variables. However, close examination revealed that this occurred since RTHvl only received direct input from LTHvl. At this point, neither of the above reasons may be ruled out.

Thirdly, a similar criticism lies in the fact that the literature does not support a direct connection between $\mathrm{LMI}_{\text {hand }}$ and contralateral secondary somatosensory area (RSII). This first-level path in the final model may also represent an indirect path modulated by an unknown ROI. A related explanation for the presence of this loading may be due to the fact that the effects of primary motor (MI) and primary sensory (SI) regions could not be dissociated. As the SII area receives both contralateral SI and SII input (Jones and Powell, 1969), the first-level path from $\mathrm{LMI}_{\text {hand }}$ to RSII could simply be due to somatosensory input distributed to both hemispheres following stimulation with TMS. Typically, the connection from MI to ipsilateral SII has been discussed in primate literature, with no mention of a connection from MI to contralateral SII (Stepniewska et al., 1993; Schmahmann and Pandya, 2006). It has not been shown that RSII is physically connected to $\mathrm{LMI}_{\text {hand }}$.

Lastly, a potential limitation of this study lies in the selection of subject data. Data were analyzed in seven subjects who showed measurable PET response during TMS (i.e., the "cortical responder" group) (Fox et al., 2004, 2006). Data were omitted from the group of "cortical non-responders" $(n=4)$, who exhibited no significant PET activations during TMS at the site of stimulation (LMI) or any other regions. When comparing the responders and non-responders, the same average EMG response was observed in terms of the magnitude and latency of response. Further analysis of these subjects indicate that the absence of a cortical response to TMS was a real effect, not an artifact of statistical thresholding (Fox et al., 2004), and it has been hypothesized that this effect was due to direct stimulation of the exiting corticospinal axons, rather than MI gray matter (Fox et al., 2004, 2006). Thus, the non-responders remain a 
perplexing group of subjects, particularly so since strong PET responses were observed during overt finger movement in this group. Inclusion of these subjects into the SEM analysis of responders would have confounded the results and rendered them difficult to interpret, since no difference in signal was detected between rest and TMS in the pre-specified ROIs. With this in mind, we chose to only analyze the responders as they revealed consistent, measurable responses during the given conditions. While it is clear that the lack of a PET-detected response during corticospinal excitation as shown by EMG is intriguing and would likely reveal connectivity differences between the responders and non-responders, this comparison was not possible due to lack of power $(n=4)$. In conclusion, the dichotomy of subject responses to TMS led to an overall bias in data selection. This selection, in turn, may possibly limit the conclusions drawn in this study, contributing to findings that cannot be extrapolated to the general population.

\section{Future work}

Applying SEM to TMS/PET data yielded insightful information on the effective connectivity of $\mathrm{LMI}_{\text {hand }}$. We feel that this technique holds great promise for further studies of effective connectivity. However, at this point, it is unclear how generalizable the results are. For example, it is unknown if any of the network connections determined in the path model are similar to the connections at work during the performance of a functional task. Future studies will involve testing the model presented in Fig. 4 in task-dependent data, such as right-handed finger tapping. Alternatively, while the model generated here included contributions from TMS data acquired over a range of intensities, this analysis could be pursued on the individual conditions, either above or below motor threshold (i.e., with and without overt movement). This type of analysis might prove valuable in dissociating the relative roles of proprioception and efference copy within the sensorimotor system. Lastly, it would be interesting to apply this exploratory analysis technique to data in which other nodes in the network were stimulated, such as the supplementary motor area, secondary somatosensory cortex, or premotor regions.

\section{Conclusions}

In this study, $\mathrm{H}_{2}^{15} \mathrm{O}$ PET data were acquired during stimulation of the hand region of the primary motor cortex $\left(\mathrm{LMI}_{\text {hand }}\right)$ using transcranial magnetic stimulation (TMS). The data were analyzed using structural equation modeling (SEM) in order to study the effective connectivity of the human motor system during taskindependent stimulation. It was determined that meta-analysis of the existing literature using activation likelihood estimation (Turkeltaub et al., 2002; Laird et al., 2005a) yielded plausible regions of the motor system in an unbiased fashion. These regions were modeled in TMS/PET data using a model-generating path analysis procedure. The resulting hierarchical model fit the data extremely well, which, based on an inspection of similar studies, may be due to the exploratory nature of the analysis. Overall, the plausibility of the brain ROIs and path connections was substantiated in the human and macaque literature. In the final path model, it was determined that the $\mathrm{LMI}_{\text {hand }}$ to right cerebellum (RCer) loading was critically important to the network interactions, despite the small path coefficient for this loading. This finding casts doubt on the practice of filtering out paths with small regression weights. Lastly, a strongly influential feedback loop was observed between $\mathrm{LMI}_{\text {hand }}$ and the cingulate gyrus (Cing).

\section{Acknowledgments}

The authors wish to thank Dr. Baxter P. Rogers of Vanderbilt University for his insightful discussions of path modeling and Suzanne T. Witt for her contributions to the finger tapping metaanalysis. This work was supported by the Human Brain Project of the NIMH (R01-MH074457-01A1; PI=Peter Fox). MDC was supported by the National Institute on Deafness and other Communication Disorders (F32DC009116).

\section{Appendix A. Instrumental variables}

To address the challenge of estimating stable and reliable structural coefficients within the context of non-recursive or interdependent systems path analytic modeling, the guidelines for using an instrumental variable (e.g., TMS intensity) were followed as offered by Heise (1975, pp. 160-161).

A. A variable, $X$ (e.g., TMS intensity), is an instrument for $Y$ (e.g., $\mathrm{LMI}_{\text {hand }}$ ) in the non-recursive relationship $Y$ (e.g., $\left.\mathrm{LMI}_{\text {hand }}\right) \rightarrow Z$ (e.g., Cing) if

(a) $X$ has no direct effect on $Z$;

(b) $X$ does affect $Y$, either directly or through an intervening variable that has no direct effect on $Z$;

(c) neither $Y$ or $Z$ has a direct or indirect effect on $X$;

(d) no unspecified factor jointly affects $X$ and $Z$ and, in general, $X$ is uncoordinated with the disturbances (i.e., errors) of $Z$.

B. A variable, $X^{\prime}$, that is only correlated with $Y$ also is an instrument for the $Y \rightarrow Z$ relationship providing it fulfills conditions (a), (c), and (d) above.

\section{Appendix B}

The application of linear structural equations to our data consisted of equations in random vectors of the form attributed to Bentler and Freeman (1983):

$\eta=\mathrm{B} \eta+\Gamma \xi+\varsigma$

where B is the square of the coefficient matrix for the structural "direct" effects of endogenous variables, $\eta$, on each other, $\Gamma$ is the coefficient matrix for the direct effects of exogenous variables, $\xi$, on endogenous variables, and $\varsigma$ is a residual error vector (Joreskog and Sorbom, 1981).

Using algebraic manipulation and substituting the right hand side of Eq. (B1) for $\eta$, we have:

$\eta=B^{2} \eta+(I+B)(\mathrm{T} \xi+\varsigma)$

Substituting (B1) into (B2), yields

$\eta=\mathrm{B}^{3} \eta+\left(\mathrm{I}+\mathrm{B}+\mathrm{B}^{2}\right)(\Gamma \xi+\varsigma)$

Performing this operation $k$ times yields

$\eta=\mathrm{B}^{k} \eta+\left(\mathrm{I}+\mathrm{B}+\mathrm{B}^{2}+\cdots+\mathrm{B}^{k-1}\right)(\Gamma \xi+\varsigma)$

In (B4), the coefficient matrix $\mathrm{B}^{k}$ represents the "indirect" effects of length $k$ for endogenous variables on each other. Since $k$ is 
arbitrary, the ultimate effect of endogenous variables on endogenous variables is given by $\mathrm{B}^{k}$ as $k$ gets arbitrarily large. The ultimate effect of exogenous variables on endogenous variables is given by $\left(I+\mathrm{B} \cdots+\mathrm{B}^{k-1}\right) \Gamma$, as $k$ gets arbitrarily large. In the case of the effects of endogenous variables on each other, the total effect is defined by the sum $\mathrm{B}+\mathrm{B}^{2}+\mathrm{B}^{3} \cdots+B^{k}$ [from (B1), (B2), (B3), and (B4)] as $k$ gets arbitrarily large. In the case of the effects of exogenous variables on endogenous variables, the total effect is defined as the ultimate effect. If these effect matrices "converge" to specific matrices, the system is termed "stable." Further, a square matrix B is called convergent (Ben-Israel and Greville, 1974) if

$\mathrm{B}^{k} \rightarrow 0 \quad$ as $\quad k \rightarrow \infty$

And a matrix is "convergent" if and only if the absolute value of the largest eigenvalue is less than 1, i.e.,

$\rho(\mathrm{B})<1$

\section{References}

Aoki, T., Tsuda, H., Takasawa, M., Osaki, Y., Oku, N., Hatazawa, J., Kinoshita, H., 2005. The effect of tapping finger and mode differences on cortical and subcortical activities: a PET study. Exp. Brain Res. 160, 375-383.

Arbuckle, J.L., 2006a. Amos (Version 7.0) [Computer Program]. SPSS, Chicago, IL.

Arbuckle, J.L., 2006b. Amos 7.0 User's Guide. SPSS, Chicago, IL.

Au Duong, M.V., Boulanouar, K., Audoin, B., Treseras, S., Ibarrola, D., Malikova, I., Confort-Gouny, S., Celsis, P., Pelletier, J., Cozzone, P.J., Ranjeva, J.P., 2005. Modulation of effective connectivity inside the working memory network in patients at the earliest stage of multiple sclerosis. NeuroImage 24, 533-538.

Ben-Israel, A., Greville, T.N.E., 1974. Generalized Inverses: Theory and Applications. Wiley, New York, NY.

Bentler, P.M., Freeman, E.H., 1983. Tests for stability in linear structura equation systems. Psychometrika 48, 143-145.

Bestmann, S., Baudewig, J., Siebner, H.R., Rothwell, J.C., Frahm, J., 2004. Functional MRI of the immediate impact of transcranial magnetic stimulation on cortical and subcortical motor circuits. Eur. J. Neurosci. 19, 1950-1962.

Blinkenberg, M., Bonde, C., Holm, S., Svarer, C., Andersen, O., Paulson, B., Law, I., 1996. Rate dependence of regional cerebral activation during performance of a repetitive motor task: a PET study. J. Cereb. Blood Flow Metab. 16, 794-803.

Bohning, D.E., Pecheny, A.P., Epstein, C.M., Speer, A.M., Vincent, D.J., Dannels, W., George, M.S., 1997. Mapping transcranial magnetic stimulation (TMS) fields in vivo with MRI. NeuroReport 8, 2535-2538.

Bollen, K., 1989. Structural Equations With Latent Variables. Wiley, New York, NY.

Brett, M., 1999. The MNI Brain and the Talairach Atlas. Cambridge Imagers, Cambridge, UK. http://www.mrc-cbu.cam.ac.uk/Imaging/mnispace. html.

Bullmore, E., Horwitz, B., Honey, G., Brammer, M., Williams, S., Sharma, T., 2000. How good is good enough in path analysis of fMRI data? NeuroImage 11, 289-301.

Calautti, C., Serrati, C., Baron, J.C., 2001. Effects of age on brain activation during auditory-cued thumb-to-index opposition: A positron emission tomography study. Stroke 32, 139-146.

Carpenter, M.B., 1985. Core Text of Neuroanatomy, 3rd ed. Williams \& Wilkins, Baltimore, MD.

Catalan, M.J., Ishii, K., Honda, M., Samii, A., Hallett, M., 1999. A PET study of sequential finger movements of varying length in patients with Parkinson's disease. Brain 122 (Pt 3), 483-495.

Cohen, J., 1988. Statistical Power Analysis for the Behavioral Sciences, 2nd ed. Lawrence Erlbaum, Inc., Hillsdale, NJ, p. 287. de Marco, G., de Bonis, M., Vrignaud, P., Henry-Feugeas, M.C., Peretti, I., 2006. Changes in effective connectivity during incidental and intentional perception of fearful faces. NeuroImage 30, 1030-1037.

Denslow, S., Lomarev, M., George, M.S., Bohning, D.E., 2005. Cortical and subcortical brain effects of transcranial magnetic stimulation (TMS)induced movement: an interleaved TMS/functional magnetic resonance imaging study. Biol. Psychiatry 57, 752-760.

Erickson, K.I., Ringo Ho, M.H., Colcombe, S.J., Kramer, A.F., 2005. A structural equation modeling analysis of attentional control: an eventrelated fMRI study. Brain Res. Cogn. Brain Res. 22, 349-357.

Fox, J., 1980. Effect analysis in structural equation models. Soc. Methods Res. 9, 3-28

Fox, P.T., Raichle, M.E., 1984. Stimulus rate dependence of regional cerebral blood-flow in human striate cortex, demonstrated by positron emission tomography. J. Neurophysiol. 51, 1109-1120.

Fox, P.T., Lancaster, J.L., 2002. Mapping context and content: the BrainMap model. Nat. Rev., Neurosci. 3, 319-321.

Fox, P., Ingham, R., George, M.S., Mayberg, H., Ingham, J., Roby, J., Martin, C., Jerabek, P., 1997. Imaging human intra-cerebral connectivity by PET during TMS. NeuroReport 8, 2787-2791.

Fox, P.T., Narayana, S., Tandon, N., Sandoval, H., Fox, S.P., Kochunov, P., Lancaster, J.L., 2004. Column-based model of electric field excitation of cerebral cortex. Hum. Brain Mapp. 22, 1-14.

Fox, P.T., Narayana, S., Tandon, N., Fox, S.P., Sandoval, H., Kochunov, P., Capaday, C., Lancaster, J.L., 2006. Intensity modulation of TMSinduced cortical excitation: primary motor cortex. Hum. Brain Mapp. 27, 478-487.

Gelnar, P.A., Krauss, B.R., Sheehe, P.R., Szeverenyi, N.M., Apkarian, A.V., 1999. A comparative fMRI study of cortical representations for thermal painful, vibrotactile, and motor performance tasks. Neuroimage 10, $460-482$

Glabus, M.F., Horwitz, B., Holt, J.L., Kohn, P.D., Gerton, B.K., Callicott, J.H., Meyer-Lindenberg, A., Berman, K.F., 2003. Interindividual differences in functional interactions among prefrontal, parietal and parahippocampal regions during working memory. Cereb. Cortex 13, $1352-1361$

Goncalves, M.S., Hall, D.A., Johnsrude, I.S., Haggard, M.P., 2001. Can meaningful effective connectivities be obtained between auditory cortical regions? NeuroImage 14, 1353-1360.

Grafton, S.T., Sutton, J., Couldwell, W., Lew, M., Waters, C., 1994. Network analysis of motor system connectivity in Parkinson's disease: modulation of thalamocortical interactions after pallidotomy. Hum. Brain Mapp. $2,45-55$.

Heise, D.R., 1975. Causal Analysis. John Wiley \& Sons, New York, NY.

Hershberger, S., Marcoulides, G., Parramore, M., 2003. In: Pugesek, B.H., Tomer, A., von Eye, A. (Eds.), Structural Equation Modeling: Applications in Ecological and Evolutionary Biology. Cambridge University Press, New York, NY, pp. 3-41.

Honey, G.D., Fu, C.H.Y., Kim, J., Brammer, M.J., Croudace, T.J., Suckling, J., Pich, E.M., Williams, S.C.R., Bullmore, E.T., 2002. Effects of verbal working memory load on corticocortical connectivity modeled by path analysis of functional magnetic resonance imaging data. NeuroImage 17, $573-582$

Ilmoniemi, R.J., Virtanen, J., Ruohonen, J., Karhu, J., Aronen, H.J., Naatanen, R., Katila, T., 1997. Neuronal responses to magnetic stimulation reveal cortical reactivity and connectivity. NeuroReport 8, 3537-3540.

Joliot, M., Crivello, F., Badier, J.M., Diallo, B., Tzourio, N., Mazoyer, B., 1998. Anatomical congruence of metabolic and electromagnetic activation signals during a self-paced motor task: a combined PETMEG study. Neuroimage 7, 337-351.

Joliot, M., Papathanassiou, D., Mellet, E., Quinton, O., Mazoyer, N., Courtheoux, P., Mazoyer, B., 1999. FMRI and PET of self-paced finger movement: comparison of intersubject stereotaxic averaged data. Neuroimage 10, 430-447.

Jones, E.G., Powell, T.P., 1969. Connexions of the somatic sensory cortex of the rhesus monkey: II. Contralateral cortical connexions. Brain 92, $717-730$ 
Joreskog, K., Sorbom, D., 1981. LISREL V. International Education Services, Chicago, IL.

Karol, E.A., Pandya, D.N., 1971. The distribution of the corpus callosum in the rhesus monkey. Brain 94, 471-486.

Kawashima, R., Inoue, K., Sugiura, M., Okada, K., Ogawa, A., Fukuda, H., 1999. A positron emission tomography study of self-paced finger movements at different frequencies. Neuroscience 92, 107-112.

Kochunov, P.V., Lancaster, J.L., Fox, P.T., 1999. Accurate high-speed spatial normalization using an Octree method. NeuroImage 10, 724-737.

Kochunov, P., Lancaster, J., Thompson, P., Toga, A.W., Brewer, P., Hardies, J., Fox, P., 2002. An optimized individual target brain in the Talairach coordinate system. NeuroImage 17, 922-927.

Laird, A.R., Fox, P.M., Price, C.J., Glahn, D.C., Uecker, A.M., Lancaster, J.L., Turkeltaub, P.E., Kochunov, P., Fox, P.T., 2005a. ALE meta-analysis: controlling the false discovery rate and performing statistical contrasts. Hum. Brain Mapp. 25, 155-164.

Laird, A.R., Lancaster, J.L., Fox, P.T., 2005b. BrainMap - the social evolution of a human brain mapping database. Neuroinformatics 3, 65-77.

Lancaster, J.L., Glass, T., B.R., L., Downs, H., Mayberg, H., Fox, P.T., 1995. A modality-independent approach to spatial normalization of tomographic images of the human brain. Hum. Brain Mapp. 3, 209-223.

Lancaster, J.L., Fox, P.T., Downs, H., Nickerson, D.S., Hander, T.A., El Mallah, M., Kochunov, P.V., Zamarripa, F., 1999. Global spatial normalization of human brain using convex hulls. J. Nucl. Med. 40, 942-955.

Lancaster, J.L., Narayana, S., Wenzel, D., Luckemeyer, J., Roby, J., Fox, P., 2004. Evaluation of an image-guided, robotically positioned transcranial magnetic stimulation system. Hum. Brain Mapp. 22, 329-340.

Lancaster, J.L., Tordesillas-Gutierrez, D., Martinez, M., Salinas, F., Evans, A., Zilles, K., Mazziotta, J.C., Fox, P.T., 2007. Bias between MNI and Talairach coordinates analyzed using the ICBM-152 template. Hum. Brain Mapp. 28, 1194-1205.

Lehéricy, S., Bardinet, E., Tremblay, L., Van de Moortele, P.F., Pochon, J.B., Dormont, D., Kim, D.S., Yelnik, J., Ugurbil, K., 2006. Motor control in basal ganglia circuits using fMRI and brain atlas approaches. Cereb. Cortex 16, 149-161.

Lerner, A., Shill, H., Hanakawa, T., Bushara, K., Goldfine, A., Hallett, M., 2004. Regional cerebral blood flow correlates of the severity of writer's cramp symptoms. Neuroimage 21, 904-913.

Mattay, V.S., Callicott, J.H., Bertolino, A., Santha, A.K., Van Horn, J.D., Tallent, K.A., Frank, J.A., Weinberger, D.R., 1998. Hemispheric control of motor function: a whole brain echo planar fMRI study. Psychiatry Res. 83, 7-22.

McIntosh, A.R., Gonzalez-Lima, F., 1994. Structural equation modeling and its application to network analysis in functional brain imaging. Hum. Brain Mapp. 2, 2-22.

McIntosh, A.R., Grady, C.L., Ungerleider, L.G., Haxby, J.V., Rapoport, S.I., Horwitz, B., 1994. Network analysis of cortical visual pathways mapped with PET. J. Neurosci. 14, 655-666.

Patel, R.S., Bowman, F.D., Rilling, J.K., 2006. A Bayesian approach to determining connectivity of the human brain. Hum. Brain Mapp. 27, $267-276$

Paus, T., Jech, R., Thompson, C.J., Comeau, R., Peters, T., Evans, A.C., 1997. Transcranial magnetic stimulation during positron emission tomography: a new method for studying connectivity of the human cerebral cortex. J. Neurosci. 17, 3178-3184.

Peltier, S., Stilla, R., Mariola, E., LaConte, S., Hu, X.P., Sathian, K., 2007. Activity and effective connectivity of parietal and occipital corti- cal regions during haptic shape perception. Neuropsychologia 45, 476-483.

Price, L.R., Tulsky, D., Millis, S., Weiss, L., 2002. Redefining the factor structure of the Wechsler Memory Scale: III. Confirmatory factor analysis with cross-validation. J. Clin. Exp. Neuropsychol. 24, 574-585.

Raftery, A., 1993. Bayesian model selection in structural equation modeling. In: Bollen, K., Long, S.J. (Eds.), Testing Structural Equation Models. Sage Publications, New Bury Park, CA, pp. 163-180.

Riecker, A., Gröschel, K., Ackermann, H., Steinbrink, C., Witte, O., Kastrup, A., 2006. Functional significance of age-related differences in motor activation patterns. Neuroimage 32, 1345-1354.

Rounis, E., Lee, L., Siebner, H.R., Rowe, J.B., Friston, K.J., Rothwell, J.C., Frackowiak, R.S., 2005. Frequency specific changes in regional cerebral blood flow and motor system connectivity following rTMS to the primary motor cortex. Neuroimage 26, 164-176.

Sadato, N., Yonekura, Y., Waki, A., Yamada, H., Ishii, Y., 1997. Role of the supplementary motor area and the right premotor cortex in the coordination of bimanual finger movements. J. Neurosci. 17, 9667-9674.

Schmahmann, J.D., Pandya, D.N., 2006. Fiber Pathways of the Brain. Oxford University Press, Oxford, NY.

Schumacker, R.E., Lomax, R.G., 2004. A Beginner's Guide to Structural Equation Modeling, 2nd ed. Lawrence Erlbaum Associates, Inc., Mahwah, NJ.

Siebner, H.R., Peller, M., Willoch, F., Auer, C., Bartnestein, P., Drzezga, A., Schwaiger, M., Conrad, B., 1999. Imaging functional activation of the auditory cortex during focal repetitive transcranial magnetic stimulation of the primary motor cortex in normal subjects. Neurosci. Lett. 270, 37-40.

Siebner, H.R., Peller, M., Willoch, F., Minoshima, S., Boecker, H., Auer, C., Drzezga, A., Conrad, B., Bartenstein, P., 2000. Lasting cortical activation after repetitive TMS of the motor cortex - a glucose metabolic study. Neurology 54, 956-963.

Speer, A.M., Willis, M.W., Herscovitch, P., Daube-Witherspoon, M., Shelton, J.R., Benson, B.E., Post, R.M., Wassermann, E.M., 2003. Intensity-dependent regional cerebral blood flow during $1-\mathrm{Hz}$ repetitive transcranial magnetic stimulation (rTMS) in healthy volunteers studied with $\mathrm{H}_{2}^{15} \mathrm{O}$ positron emission tomography: II. Effects of prefrontal cortex rTMS. Biol. Psychiatry 54, 826-832.

Stepniewska, I., Preuss, T.M., Kaas, J.H., 1993. Architectonics, somatotopic organization, and ipsilateral cortical connections of the primary motor area (M1) of owl monkeys. J. Comp. Neurol. 330, 238-271.

Talairach, J., Tournoux, P., 1988. Co-Planar Stereotaxic Atlas of the Human Brain. Thieme, New York, NY.

Taniwaki, T., Okayama, A., Yoshiura, T., Togao, O., Nakamura, Y., Yamasaki, T., Ogata, K., Shigeto, H., Ohyagi, Y., Kira, J., Tobimatsu, S., 2006. Functional network of the basal ganglia and cerebellar motor loops in vivo: different activation patterns between self-initiated and externally triggered movements. NeuroImage 31, 745-753.

Turkeltaub, P.E., Eden, G.F., Jones, K.M., Zeffiro, T.A., 2002. Meta-analysis of the functional neuroanatomy of single-word reading: Method and validation. NeuroImage 16, 765-780.

Yoo, S.S., Wei, X., Dickey, C.C., Guttmann, C.R., Panych, L.P., 2005. Longterm reproducibility analysis of fMRI using hand motor task. Int. J. Neurosci. 115, 55-77.

Zheng, X.B., Rajapakse, J.C., 2006. Learning functional structure from fMR images. NeuroImage 31, 1601-1613.

Zhuang, J.C., LaConte, S., Peltier, S., Zhang, K., Hu, X.P., 2005. Connectivity exploration with structural equation modeling: an fMRI study of bimanual motor coordination. NeuroImage 25, 462-470. 